\section{EFSUMB Newsletter}

\section{European Federation of Societies for Ultrasound in Medicine and Biology}

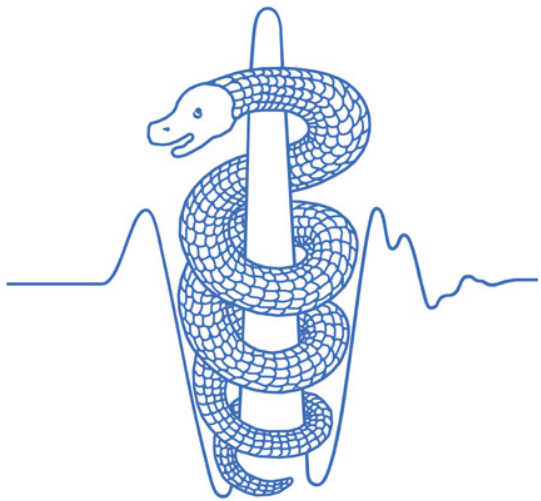

\title{
Ultrasound handheld devices - potential problems and possible solutions
}

Most ultrasound (US) manufacturers offer handheld devices in addition to their more traditional larger mid- and high-end machines. The price of handheld devices is generally lower, but they may lack features like elastography, contrast agent imaging and biopsy guidance. An advantage of handheld US devices is that due to their small size they fit into the pockets of a physician's lab coat. The smallest handheld devices are composed of a transducer with an USB connection to a tablet or a smartphone. This means that handheld devices could be available to the physician at all times while on duty. But is this setup sufficient for diagnostic use [1]?

Let us first take a short look at the monitors used for radiographs in radiology. There are several requirements for a diagnostic monitor, including pixels per inch, resolution, bit depth, luminance, contrast and brightness. In addition, there will also be strict requirements for calibration and quality control. Such requirements do not exist for handheld devices, which may vary in image quality [2] and they may not be suitable for diagnostics, although ne-

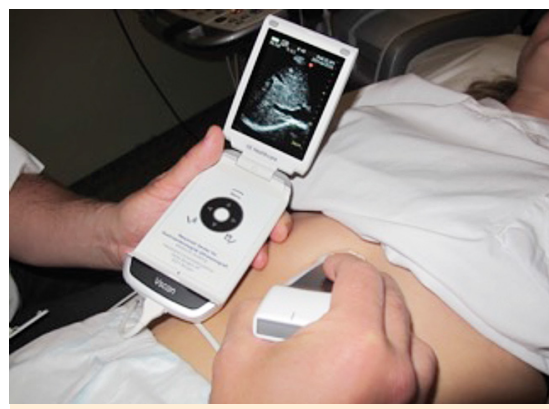

Just one example of the many handheld devices available wer high-end tablets and smartphones will offer full HD or even quad-HD displays and should therefore not lack in pixels but a more careful examination of the other specifications could mean they are not suitable for diagnostics in radiographs.

Radiographs are stored in the DICOM format and several viewers are now available as apps. Many PACS/DICOM software solutions also offer a tablet viewer, e.g. OsiriX (Pixmeo, Switzerland), but there are different local legal aspects limiting their use.

CT, MRI and US images will have a smaller image matrix than radiographs and could therefore most likely be displayed sufficiently on tablets or smartphones. Several papers on the use of iPads for limited diagnostic questions in CT or MRI have been published showing the potential [3-7], and in a systematic review, the use of a tablet was appropriate for interpretation of radiographs for an on-call radiologist [8].

To our knowledge similar requirements for tablets and smartphones used for displaying ultrasound images are yet to be suggested.

Point-of-care ultrasound and emergency medicine are areas where handheld US devices could prove useful and where the lack of high-end features of the device will not be a limitation. In other medical fields the lack of optimal conditions for performing US may limit the value of the examination. For instance, the best discrimination of grey scale pixels requires a dark room and the reflectivity of the screen of the device may also limit the examination.
A good patient contact would also require some privacy, which should be ensured in all ultrasound examinations.

Obstetrics could also be a preferential field for the application of handheld devices, both in the emergency room (e.g. for the documentation of fetal viability or placental position and state) and in the labour ward (e. g. for the determination of fetal lie and progress of labour). It is therefore striking that there is currently only one study comparing measurement of the abdominal circumference using a handheld device with fundal height measurement [9].

Pre-hospital ultrasound with handheld devices has been available for the Norwegian air ambulance doctors for more than one year. The handheld devices are used to support clinical decision making under transport. Particularly clinical situations with dyspnea, free abdominal fluid and cardiac arrest, pocket size US can provide vital clinical information, and change the treatment and even the hospital of destination. A two day introductory course has been offered, and there is a need for follow up courses by the doctors who have taken up the use of handheld US. There is still no obligation for air ambulance personnel to use US in their practice, and there are several challenges in image storing and transfer which may be overcome with technical development.

\section{EFSUMB}

Lynne Rudd

28 Portland Place, London

W1B 1LY, United Kingdom

Tel: +44 (0) 2070997140

Email: efsumb@efsumb.org 
A systematic review of the use of portable ultrasound devices in low- and middleincome countries found most applications focused on obstetrical and abdominal complaints [10]. Some described that portable ultrasound may have an impact on clinical management in up to $70 \%$ of all cases. However, the quality of the current evidence was low [10] and larger clinical trials are needed. One study found that overall fewer diagnoses could be made with a handheld US device compared to using a high-end machine but it was sufficient for distinct pathology like ascites [11].

A recent study found that pocket-sized ultrasound showed poor diagnostic accuracy and confidence when given to internal medicine residents after a 3 hour training session and 1 month of independent practice, highlighting the need for proper training [12].

Training of every medical doctor to perform ultrasound examinations is a huge task and we need appropriate training methods, which are not just based on a fixed number of patients to be examined but on a skill-based system possibly using simulators for this to be successful $[13,14]$. Also, it would most likely be necessary to introduce US at least on a basic level in the curriculum for medical students $[15,16]$.

Michael Bachmann Nielsen ${ }^{1}$,

Roald F. Havre ${ }^{2}$,

Caroline Ewertsen ${ }^{1}$,

Vito Cantisani ${ }^{3}$,

Maija Radzina ${ }^{4}$,

Alexandros Sotiriadis ${ }^{5}$

${ }^{1}$ Department of Radiology, Rigshospitalet, Copenhagen, Denmark

${ }^{2}$ Department of Medicine, National Centre for Ultrasound in Gastroenterology, Haukeland University Hospital, Bergen, Norway

${ }^{3}$ Department of Radiological Sciences, Univ. Sapienza, Rome, Italy

${ }^{4}$ Pauls Stradins Clinical University Hospital, Institute of Diagnostic Radiology, Riga, Latvia

${ }^{5}$ Obstetrics and Gynecology, Aristotle

University of Thessaloniki, Greece
References

1 Christoph F Dietrich et al. Birth of Echoscopy - the EFSUMB Point of View. Ultraschall in Med 2013; 34(1): 92

2 Yamazaki A, Liu P, Cheng WC, Badano A. Image quality characteristics of handheld display devices for medical imaging. PLoS One 2013; 8(11): e79243

3 Johns S, Poh AC, Lim TC, Chan EH, Chong IR. The iPad tablet computer for mobile on-call radiology diagnosis? Auditing discrepancy in CT and MRI reporting. J Digit Imaging 2012; 25; 628-34

4 Schlechtweg PM, Kammerer FJ, Seuss H, Uder $M$, Hammon $M$. Mobile Image Interpretation: Diagnostic Performance of CT Exams Displayed on a Tablet Computer in Detecting Abdominopelvic Hemorrhage. J Digit Imaging. 2016; 29: 183-8.

5 Tadinada A, Mahdian M, Sheth S, Chandhoke TK, Gopalakrishna A, Potluri A, Yadav S. The reliability of tablet computers in depicting maxillofacial radiographic landmarks. Imaging Sci Dent. 2015; 45: 175-80

6 Hammon M, Schlechtweg PM, Schulz-Wendtland $R$, Uder $M$, Schwab SA. iPads in Breast Imaging - A Phantom Study. Geburtshilfe Frauenheilkd. 2014; 74(2): 152-156

7 Zwart CM, He M, Wu T, Demaerschalk BM, Mitchell JR, Hara AK. Selection and pilot implementation of a mobile image viewer: a case study.

8 Caffery LJ, Armfield NR, Smith AC. Radiological interpretation of images displayed on tablet computers: a systematic review. Br J Radiol. 2015; 88(1050): 20150191

9 Haragan AF, Hulsey TC, Hawk AF, Newman RB, Chang EY. Diagnostic accuracy of fundal height and handheld ultrasound-measured abdominal circumference to screen for fetal growth abnormalities. Am J Obstet Gynecol. 2015; 212(6): 820.e1-8
10 Becker DM, Tafoya CA, Becker SL, Kruger GH, Tafoya MJ, Becker TK. The use of portable ultrasound devices in low- and middle-income countries: a systematic review of the literature. Trop Med Int Health. 2016; 21(3): 294-311

11 Stock KF, Klein B, Steubl D, Lersch C, Heemann $U$, Wagenpfeil S, Eyer F, Clevert DA. Comparison of a pocket-size ultrasound device with a premium ultrasound machine: diagnostic value and time required in bedside ultrasound examination. Abdom Imaging. 2015 Oct; 40(7): 2861-6

12 Ojeda JC, Colbert JA, Lin X, McMahon GT, Doubilet PM, Benson CB, Wu J, Katz JT, Yialamas $M A$. Pocket-sized ultrasound as an aid to physical diagnosis for internal medicine residents: a randomized trial. J Gen Intern Med. 2015; 30(2): 199-206

13 Konge L, Albrecht-Beste E, Nielsen MB. Virtual-reality simulation-based training in ultrasound. Ultraschall in Med. 2014; 35(2): 95-7

14 Østergaard ML, Ewertsen C, Konge L, AlbrechtBeste E, Bachmann Nielsen M. SimulationBased Abdominal Ultrasound Training - A Systematic Review. Ultraschall in Med 2016; Feb 16 (Epub ahead of print)

15 Cantisani V, Dietrich CF, Badea R, Dudea S, Prosch H, Cerezo E, Nuernberg D, Serra AL, Sidhu PS, Radzina M, Piscaglia F, Bachmann Nielsen M, Calliada F, Gilja OH. EFSUMB statement on medical student education in ultrasound [short version]. Ultraschall in Med. 2016; 37(1): 100-102

16 Konge L, Albrecht-Beste E, Bachmann Nielsen $M$. Ultrasound in Pre-Graduate Medical Education. Ultraschall in Med. 2015; 36: 213-5 